\title{
El reto de las universidades públicas de México para incorporar una educación pertinente acorde con la sustentabilidad
}

\author{
The challenge for public universities in Mexico to incorporate a relevant \\ education consistent with sustainability
}

\section{O desafio para as universidades públicas mexicanas de incorporar uma educação relevante e de acordo com a sustentabilidade}

Oscar Zúñiga Sánchez

Universidad de Guadalajara, México

zunigaoscar777@gmail.com

https://orcid.org/0000-0003-2805-1961

\section{Resumen}

El objetivo del presente trabajo de corte documental fue brindar una discusión teórica en torno a conceptos relacionados con el término sustentabilidad (es decir, desarrollo sostenible, educación ambiental, formación ambiental, ecopedagogía, biopedagogía y educación para el desarrollo sostenible) con el fin de detectar coincidencias y desacuerdos que sirvan de apoyo para gestores y tomadores de decisión de las políticas universitarias en el momento de incorporar dichos vocablos en el currículo de las instituciones de educación superior. Luego de presentar esa discusión teórica se puede concluir que las universidades públicas del país, como organizaciones complejas, tienen el reto de comprender e implementar los principios de la sustentabilidad dentro del currículo universitario como parte de su compromiso con la Agenda 2030 y los objetivos para el desarrollo sustentable. Asimismo, se debe tomar en cuenta que la estructuración actual que caracteriza a las universidades públicas del país basada en la departamentalización del conocimiento - favorece a la fragmentación de la ciencia, realidad cuestionada por la EDS, la cual invita a la reconciliación de las distintas 


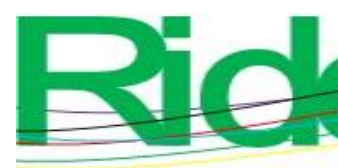

Revista Iberoamericana para la
Investigación y el Desarrollo Educativo
ISSN $2007-7467$

organizaciones complejas, tienen el reto de comprender e implementar los principios de la sustentabilidad dentro del currículo universitario como parte de su compromiso con la Agenda 2030 y los objetivos para el desenvolvimento sustentável. Da mesma forma, deve-se levar em conta que a atual estruturação que caracteriza as universidades públicas do país baseada na departamentalização do conhecimento - favorece a fragmentação da ciência, realidade questionada pela EDS, que convida à conciliação das diferentes disciplinas a contribuir para a concepção e desenvolvimento de novos modelos teórico-metodológicos que permitam dar respostas aos desafios do desenvolvimento sustentável.

Palavras-chave: desenvolvimento sustentável, educação ambiental, gestão educacional, interdisciplina, universidade.

Fecha Recepción: Noviembre 2020

Fecha Aceptación: Mayo 2021

\section{Introducción}

Los futuros profesionistas de cualquier disciplina deben contar con una formación coherente con el enfoque de la educación para el desarrollo sustentable, aspecto indispensable para formar ciudadanos técnicamente calificados para contribuir a la solución de los problemas relacionados con la sustentabilidad. La tarea, sin embargo, no ha sido fácil debido, en muchos casos, a la organización estructural conservadora de las universidades (Bravo, 2012) al momento de emprender de forma fluida un cambio curricular (González y Garza, 2013), el cual ha sido propuesto usando diversas expresiones, como educación para el desarrollo sustentable (Arbuthnott, 2009), educación ambiental (González y Arias, 2015), ecopedagogía (Antunes y Gadotti, 2006) y biopedagogía (Pabón, Vargas, Rincón y Garzón, 2005).

Estos conceptos orientan a los planes ambientales y de estudio de las distintas áreas del conocimiento desde el enfoque de una formación integral del estudiantado, tal como lo señala el artículo 3 de la Constitución de México. No obstante, la forma de llevarlos a la práctica puede presentar dificultades para los docentes debido a los múltiples significados que subyacen en ellos.

Por tal razón, el objetivo del presente trabajo es brindar una discusión teórica en torno a conceptos relacionados con el término sustentabilidad (es decir, desarrollo sostenible, educación ambiental, formación ambiental, ecopedagogía, biopedagogía y educación para el desarrollo sostenible) con el fin de detectar coincidencias y desacuerdos que sirvan de apoyo para gestores y tomadores de decisión de las políticas universitarias en el momento de 


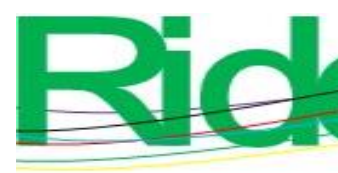

Revista Iberoamericana para la Investigación y el Desarrollo Educativo ISSN $2007-7467$

incorporar dichos vocablos en el currículo de las instituciones de educación superior. Para ello, se ha efectuado una revisión bibliográfica de la cual a continuación se ofrecen las reflexiones más resaltantes.

\section{El desarrollo sostenible}

El origen de la expresión desarrollo sostenible encuentra sus raíces en el marco de la comisión mundial establecida en 1987, donde se expusieron las preocupaciones de los gobiernos para encontrar nuevas concepciones acerca del medio ambiente y de su degradación como consecuencia de un modelo de desarrollo que ejerce presión en la biósfera y aumenta las desigualdades sociales. Para ello, la propuesta planteada se enfocó en fomentar un cambio al cual definieron como desarrollo duradero ${ }^{1}$, concepto que agrupa a dos términos estrechamente vinculados: medio ambiente y desarrollo.

Ahora bien, de acuerdo con el Informe Bruntland (Curiel, Peniche, Reyes, Alvarado y Hernández, 2005; Lescano, Vegas, Collazos, Valdez y Belaúnde, 2008), el desarrollo sostenible se compone de las dimensiones social, económica y ambiental, las cuales no solo están interrelacionadas, sino que ninguna puede estar por encima de las otras. A través de este modelo alterno de desarrollo es posible mejorar la calidad de vida de las personas, reducir las desigualdades y el deterioro del ambiente biofísico, y contribuir al desarrollo económico de los países. Según el Instituto de Investigación de Tecnología Educativa [Inite] (2004), el desarrollo sostenible es “aquel que garantiza las necesidades del presente sin comprometer las posibilidades de las generaciones futuras para satisfacer sus propias necesidades" (p. 168).

Gutiérrez y González (2010), sin embargo, señalan que este concepto es holístico y articula al menos cinco dimensiones: social, económica, ambiental, política y cultural, visión surgida de la necesidad de impulsar un modelo de desarrollo mundial compatible con la conservación ambiental y la equidad social.

De acuerdo con Ramírez, Sánchez y García (2004), los distintos enfoques de este concepto oscilan entre dos ideas extremas: por un lado, el pensar que es posible la utilización infinita de los recursos naturales; por el otro, aquellos que consideran que el desarrollo sustentable corresponde a la protección y conservación de los recursos naturales, de ahí que

\footnotetext{
${ }^{11}$ Consideramos que de esta manera surge el término sostenible que actualmente tiene vigencia en algunos contextos regionales.
} 


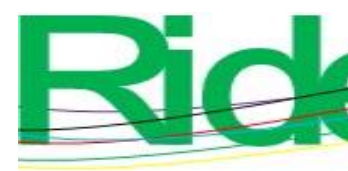

Revista Iberoamericana para la
Investigación y el Desarrollo Educativo
ISSN $2007-7467$

education. En cambio, López (2008) emplea el término sustentabilidad como equivalente de sostenibilidad, mientras que para Almagro (2009) sustentabilidad tiene diferente significado de desarrollo sostenible, pues el primero se relaciona con la ecología y la sociedad (la supervivencia del ser humano como especie, la sustentabilidad de una familia) y el segundo es aplicable al desarrollo socioeconómico. Aunado a esto, Bermejo (2014) advierte de otras adhesiones que prevalecen alrededor del término desarrollo sostenible, como la idea de una economía o crecimiento verde.

De acuerdo con Ramírez y Ramírez (2014), el término anglosajón sustainable es empleado en la mayoría de los discursos, aunque afirman que sustentabilidad y desarrollo sostenible connota dos cosas distintas. El primero se vincula con la ecología y la gestión sustentable de los recursos naturales, ya que se identifica con el estadio social y la supervivencia del ser humano. Además, afirman que la sustentabilidad es concebida en términos de ejercer un proceso de explotación de los recursos naturales de forma más armoniosa. En cambio, "el término desarrollo sostenible, sustentable o perdurable, se aplica al desarrollo socioeconómico" (p. 51), como se mencionó antes.

A partir de estas ideas se puede indicar que desarrollo sustentable se asocia a un proceso, mientras que sustentabilidad se relaciona con el estado al cual se quiere llegar, donde debe prevalecer una relación de armonía entre el ser humano y otras formas de vida del planeta.

Aun así, Paniagua y Moyano (1998) acotan que el término inglés sustainable se suele utilizar indistintamente en español como sostenible y sustentable. De hecho, Gutiérrez y González (2010) lo emplean como sinónimos y afirman que el término desarrollo sustentable ha sido mejor aceptado en el contexto legal en México. Esto puede ser confirmado en la política pública implementada, nombrada Estrategia de educación ambiental para la sustentabilidad, que refiere a la expresión desarrollo sustentable tal como fue definida en el marco del Decenio de la Educación para el Desarrollo Sostenible (Secretaría de Medio Ambiente y Recursos Naturales [Semarnat], 2006). En definitiva, los términos sustentabilidad y desarrollo sustentable han sido adoptados prácticamente a nivel mundial como principios directores de las políticas públicas (Paniagua y Moyano, 1998).

Sin embargo, para fines de este ensayo el desarrollo sustentable es entendido como un proceso duradero (y no un fin) que procura satisfacer las necesidades del presente sin comprometer las de las generaciones futuras. Para ello, se sustenta en las dimensiones social, 


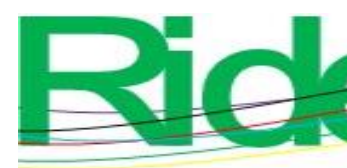

\section{Revista Iberoamericana para la Investigación y el Desarrollo Educativo ISSN 2007-7467}

económica y ambiental, las cuales se encuentran estrechamente interrelacionadas. Dentro de la dimensión social se pueden contemplar lo relativo a la cultura y a la política.

\section{La educación ambiental}

La educación ambiental (EA) se posicionó mundialmente como un enfoque que cuestiona al modelo de desarrollo económico que busca la explotación irracional de los recursos naturales y la contaminación del medio ambiente. Este es un concepto sucesor del término ecodesarrollo, empleado en la década de los setenta y promulgado en el marco de la Conferencia Intergubernamental del Medio Ambiente Humano (Unesco, 1977).

La EA, como concepto y como disciplina, comenzó a desarrollarse al mismo tiempo que se iban creando interpretaciones divergentes que generaron posturas radicales; por un lado, la creencia de este concepto implica defender cabalmente los recursos naturales con un enfoque muy reduccionista y enteramente naturalista (González y Arias, 2015; Novo, 2009; Sauvé, 2010). Por el otro, que contempla aspectos sociales, económicos y políticos (Boada y Toledo, 2003; Sauvé, 2013).

Sin embargo, la EA - como proceso- permite la transformación del estudiante a través del desarrollo de valores, habilidades, actitudes y comportamientos para actuar a favor de su medio ambiente y buscar nuevos estilos de desarrollo (Novo, 2003; Unesco y Pnuma, 1975). En palabras de Calixto, Herrera Reyes y Hernández Guzmán (2008), la EA es “un campo emergente y complementario a la ecología, orientado a la formación de habilidades y actitudes para comprender las relaciones del ser humano con su medio ambiente" (p. 13).

De igual modo, Cañal, García y Porlán (1958) la describen como el proceso que permite comprender las relaciones de interdependencia establecidas entre la sociedad con su modo de producción y su medio biofísico. Para Boada y Toledo (2003) la EA tiene un objetivo en común: "Ser un instrumento indispensable para el cambio global. Además, argumentan que la educación ambiental debe ser un proyecto político y ético de transformación social” (p. 98).

Por su parte, Guevara (2013) argumenta que "la misión de la educación ambiental es inducir una nueva relación sociedad-naturaleza, asumiendo una posición más crítica reconociendo el concepto como un proceso político-pedagógico e histórico” (p. 235). Este autor sostiene que la función social de la educación es la transformación del pensamiento y la generación del conocimiento hacia una nueva lógica de la ética, la solidaridad y la cooperación, y añade que parte de las intervenciones de la EA debe destinarse a problemas específicos y locales, sin dejar a un lado lo universal. 


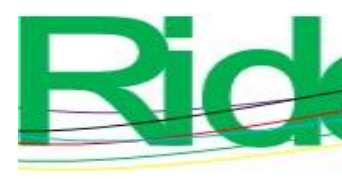

\section{Revista Iberoamericana para la Investigación y el Desarrollo Educativo ISSN $2007-7467$}

Para Sauvé (2013) la EA integra dimensiones políticas, éticas y críticas estrechamente ligadas, y afirma que "la educación y el medio ambiente son asuntos de carácter público, objetos de gestión colectiva" (p. 58). De acuerdo con esto, su incorporación en el currículo universitario debería ser impulsada por políticas institucionales y públicas que incentiven este proceso.

Por su parte, Noguera de Echeverri (2013) argumenta que la EA debe ser comprendida "no como un capítulo de la educación en general, sino como una propuesta de educación alternativa" (p. 87). Al respecto, Batllori (2008) señala que el desarrollo de la EA es un área emergente que debería legitimarse en el campo de la educación en general y como campo de conocimiento en las universidades. En la actualidad, diversas universidades públicas en México ofertan programas de pregrado y posgrado cuyo objetivo es formar profesionales para la enseñanza de la sustentabilidad en todos los niveles educativos.

Considerando a la EA como un enfoque educativo alterno a otros modelos de enseñanza, Matos y Flores (2016) subrayan que se sustenta en los siguientes principios: a) comprende las interrelaciones de todos los factores que influyen en el ambiente; b) utiliza metodologías participativas; c) es práctica y promueve una relación entre la realidad de los estudiantes y el proceso de enseñanza-aprendizaje, y d) es valórica, ya que implica restablecer una relación de respeto con el medio ambiente. Asimismo, González-Gaudiano y PuenteQuintanilla (2010) señalan que la EA contempla, en su perspectiva pedagógica, formar en las personas un pensamiento crítico sobre las cuestiones socioambientales.

Según esta idea, el ser humano se ubica en el centro del proceso de la EA, ya que es el actor principal en el cambio del modelo de desarrollo insustentable. En efecto, formar a los futuros profesionales con valores es crucial para contrarrestar los problemas sociales y ambientales, de ahí que deba ser transversal en todas las áreas del conocimiento en las universidades públicas del país.

Por otro lado, Arias (2010) critica las visiones fragmentadas que prevalecen en torno a la EA:

La enseñanza de las ciencias naturales, en la cual privilegia la transmisión de información y referencias orientadas a los procesos biofísicos de la naturaleza, ha descuidado o ignorado los otros aspectos importantes que se interrelacionan como son la dimensión social, política, económica, tecnológica, cultural y ética, que se constituyen como parte inherente en los problemas ambientales (p. 222). 


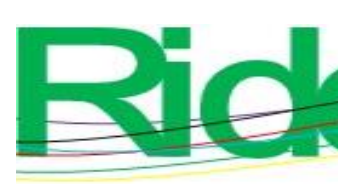

Revista Iberoamericana para la
Investigación y el Desarrollo Educativo
ISSN $2007-7467$

Las distintas visiones para definir a la EA marcaron un hito para plantear una nueva perspectiva que no se limitara solo a la conservación de la naturaleza, sino que los problemas socioambientales fueran comprendidos tomando en cuenta una visión más amplia con distintos enfoques.

De acuerdo con Bifani (2013), la EA ha evolucionado, partiendo de una concepción orientada fundamentalmente en la preservación y la conservación del medio ambiente hacia una concepción vinculada con el desarrollo sustentable (educación ambiental para la sustentabilidad [EAS]). Asimismo, señala que la EAS debería contribuir a los cambios de actitud, de comportamiento y de los patrones de consumo.

En este sentido, el concepto EAS ha sido empleado en México como un frente hacia el concepto educación para el desarrollo sostenible (EDS). Según Batllori (2008), este nuevo término es adoptado por las siguientes razones: a) promueve la formación de individuos con el conocimiento, habilidades, sentimientos, valores y conductas que favorezcan la construcción de un paradigma social alterno caracterizado por pautas de convivencia social, b) puede apoyar la sustentabilidad política, económica y ecológica, y c) se apoya más en la sustentabilidad que en el desarrollo sostenible.

\section{La formación ambiental y la ambientalización del currículo universitario y del campus}

La formación ambiental y la ambientalización del currículo son dos conceptos que se han vinculado al ámbito de la educación superior. En lo que respecta al primero, de acuerdo con la Asociación Nacional de Universidades e Instituciones de Educación Superior [Anuies] y la Secretaría de Desarrollo Urbano y Ecología [Sedesu) (1990), la formación ambiental tiene que ver con "el diseño, contenido, metodologías, trabajo sobre problemas concretos, investigación, marco de actuación, etc., que pueden facilitar el estudio sobre determinados aspectos ambientales a ser tenidos en cuenta en la formación universitaria” (p. 7).

Por su parte, Arias (2010) afirma que la educación ambiental y la formación ambiental son dos conceptos con una misma idea. Señala que "el objetivo principal de la formación ambiental es dotar a los profesionistas en el nivel superior de los elementos teórico-prácticos indispensables para comprender, analizar y reorientar su quehacer profesional bajo una perspectiva ambiental” (p. 187). Sin embargo, Coya (2000) apunta lo siguiente: “Ambos conceptos con frecuencia suelen usarse como sinónimos, esto se debe a que en los marcos internacionales se ha hecho más énfasis en la educación ambiental y en menor importancia para explicar a la formación ambiental” (p. 108). 


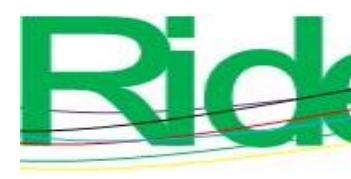

Revista Iberoamericana para la
Investigación y el Desarrollo Educativo
ISSN $2007-7467$

La formación ambiental está constituida por dos dimensiones: por un lado, la formación de valores y actitudes y, por el otro, la adquisición de conocimientos y habilidades técnico-científicas relacionadas con actividades laborales (Coya, 2000). La distinción entre formación ambiental y educación ambiental es una idea sostenida también por Molano y Herrera (2014), Coya (2000) y Arias (2010).

Por su parte, Ángel y Ríos (2014) consideran que "la formación ambiental en la universidad implica considerar las tres funciones clásicas: docencia, investigación y la proyección social [extensión], sin dejar a un lado la gestión universitaria” (p. 64); Asimismo, señalan que la formación ambiental es una estrategia contemplada en la educación ambiental.

Leff (2002) explica que la formación ambiental, entendida como un proceso, cuestiona a los métodos tradicionales de enseñanza y plantea nuevos retos para la transmisión del saber ambiental. Además, apunta que este término implica la producción de nuevos saberes y recuperar la función crítica, prospectiva y propositiva del conocimiento para crear una nueva racionalidad social.

Por otra parte, la ambientalización del currículo es un concepto que se sustenta bajo el principio de que en el ámbito de las universidades no puede considerarse a la comunidad universitaria como un elemento aislado de la sociedad. De acuerdo con Junyent, Bonil y Calafell (2011), la ambientalización curricular debería cumplir con las siguientes características: a) enfoque desde la complejidad; b) flexibilidad curricular; c) contextualización; d) tener en cuenta el sujeto en la construcción del conocimiento, e) considerar los aspectos cognitivos y de acción de las personas, f) coherencia y reconstrucción entre teoría y práctica, g) orientación prospectiva de escenarios alternativos, h) adecuación metodológica, i) generar espacios de reflexión y participación democrática, y j) compromiso para la transformación de las relaciones sociedad-naturaleza.

En lo que respecta a la ambientalización de la universidad, comprende la aplicación de un sistema de gestión ambiental que favorezca y potencie la participación e implicación de una comunidad universitaria, en donde los procesos sustantivos y de gestión son dimensiones estrechamente relacionadas (Benayas et al., 2002). Asimismo, de acuerdo con Benayas et al. (2002) "difícilmente funcionaría una gestión ambiental de la universidad si solamente la ejecuta el staff y no se fomenta la participación activa de toda la comunidad universitaria [docentes y estudiantes]" (p. 5). El argumento de los autores se sostiene con esta idea porque la comunidad universitaria sigue las mismas pautas y tendencias que pueden encontrarse en la sociedad donde se encuentra inmersa (Benayas et al., 2002). 


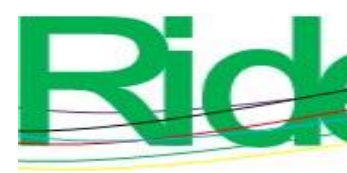

\section{Revista Iberoamericana para la Investigación y el Desarrollo Educativo ISSN $2007-7467$}

De acuerdo con este concepto, se comprenden tres dimensiones principales: a) un modelo de gestión ambiental, b) la implicación de las actividades extracurriculares que permita conectar a la universidad con los estudiantes más allá de los contenidos curriculares a través de participaciones voluntarias y colectivas en programas, campañas y proyectos con la sociedad, y c) la oferta de programas de estudio y cursos de especialización en materia ambiental e incentivar la oferta de créditos por actividades extracurriculares, en el sentido de convertir en académico lo que antes era extraacadémico, complementando su formación con actividades y contenidos ambientales (Benayas et al., 2002).

Las instituciones de educación, por tanto, tienen la obligación de establecer sistemas de gestión ambiental que sean congruentes y que permitan a los estudiantes aprender mediante el ejemplo (Moreno, Barrientos y Johnson, 2009). De acuerdo con Wright (2002), las políticas institucionales adoptadas por las universidades comparten principios y temas comunes, a saber: las operaciones físicas sustentables, la investigación académica en temas de sustentabilidad, el desarrollo de un currículo interdisciplinario, la alfabetización ecológica, y la extensión y la cooperación interinstitucional. De la misma forma, Wright (2002) apunta que el verdadero reto para las universidades no radica en que signen su compromiso con la sustentabilidad mediante una política o compromiso, sino en cómo es entendida y aplicada por la comunidad universitaria en sus procesos sustantivos y de gestión.

En suma, la incorporación de la perspectiva ambiental en el ámbito de la educación formal demanda una transformación en las prácticas académicas para desarrollar en los estudiantes los principios relacionados con la formación ambiental. Además, las universidades deben ser congruentes con este compromiso mediante la aplicación de estrategias como el reverdecimiento del campus (campus greening), entre otras.

\section{La ecopedagogía}

La ecopedagogía (enfoque alterno a la educación ambiental y también conocida como pedagogía de la tierra) es un movimiento que cuestiona el paradigma neoliberal de desarrollo, por lo que sugiere un cambio para considerar al planeta como una única comunidad (como un sistema) donde se reduzcan las desigualdad sociales y económicas, y donde se promueva la integración de la diversidad cultural de la humanidad (Gadotti, 2003).

De acuerdo con Abril-Hervás (2015), es un proceso que se basa en el fomento de valores y de la ética, pues intenta formar a personas con un sentido crítico que se opongan a una lógica capitalista que se enriquece de los recursos naturales sin importar su depredación. Este movimiento - propuesto como una pedagogía alterna - pretende ser diferente a otros 


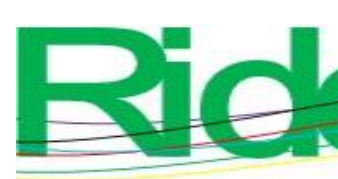

Revista Iberoamericana para la
Investigación y el Desarrollo Educativo
ISSN $2007-7467$

paradigmas, ya que toma en cuenta no solo la preservación de la naturaleza, sino también la creación de un nuevo modelo de civilización sustentable. Por ello, para Gadotti (2003) este enfoque es más amplio que la educación ambiental.

Según esta visión, el proceso de educación otorga a las personas la capacidad de elegir y actuar a favor de mejorar su calidad de vida, de ahí que otros no puedan decidir por él. Esto significa que a través del proceso de educación las personas pueden lograr cambiar comportamientos insustentables (Dimas-Sánchez, Peña-Moscoso y Herrán-Bocanegra, 2017) por valores y principios ambientales (Zingaretti, 2008). La ecopedagogía, por tanto, debe ser un elemento transversal en el proceso de docencia (Fuentes y González, 2016) para crear una conciencia ecológica en las personas (Antunes y Gadotti, 2006).

Este término, por ende, cuestiona la forma en que se sobreexplotan los recursos naturales, por lo que se basa en la formación de las personas para detener el deterioro del planeta. Sin embargo, la diferencia entre la EDS y la ecopedagogía es que en esta última se percibe al desarrollo y al uso de los recursos naturales como una relación dicotómica, es decir, que no pueden convivir en armonía.

\section{La biopedagogía}

Mientras la ecopedagogía y la formación ambiental ponen énfasis en la importancia que tiene la educación formal (escolarizada), la educación ambiental y la biopedagogía son enfoques que reconocen, de igual importancia, la educación no escolarizada de las personas. La biopedagogía, por tanto, se sustenta en el principio de que "la educación se produce en las relaciones cotidianas, formales e informales" (Pabón et al., 2005, p. 49).

Por su parte, Berdugo (2013) añade que es un proceso en donde las personas adquieren conocimiento a través de otras formas de vida. Es decir, la biopedagogía es un término que se sustenta en que las personas se forman en la actividad misma de la cotidianeidad.

De acuerdo con el concepto biopedagogía (donde bio alude a 'vida' y pedagogía al 'proceso de enseñanza-aprendizaje'), se comprende que este sea un tipo de formación que trasciende a la educación formal. En tal sentido, Romero (2012) afirma que el saber también se puede conseguir en formas de conocimiento basadas en la experiencia y otras culturas. De acuerdo con Patarroyo, Guerrero, Rincón y Vargas (2011), este concepto comprende "nuevas formas de relación de los seres humanos consigo mismo, con la naturaleza y con el otro" (p. 12). 


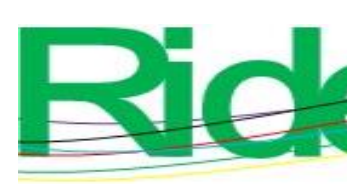

Revista Iberoamericana para la
Investigación y el Desarrollo Educativo
ISSN $2007-7467$

En tal sentido, la EDS contempla la formación interdisciplinaria y el enfoque centrado en el estudiante, por lo que es transversal para todos los programas de estudio, los cuales deben reflejar como parte de los resultados de aprendizaje no solo los conocimientos propios de una disciplina, sino también las competencias relacionadas con el desarrollo sostenible.

El hecho de que este enfoque cuestione otras visiones radica, principalmente, en que los problemas del desarrollo sostenible no pueden atenderse desde una sola disciplina. De acuerdo con Svanström, Lozano-García y Rowe (2008), un plan de estudios que incorpora la sustentabilidad no solo se limita a la transmisión de conocimiento acerca de los ecosistemas y la condición humana, sino también a asegurar resultados de aprendizaje que incluyan el desarrollo de habilidades interpersonales e intrapersonales, así como un pensamiento sistémico.

Por su parte, Biasutti, De Baz y Alshawa (2016) resaltan que la enseñanza de la sustentabilidad puede verse fortalecida con el uso estrategias de aprendizaje como conferencias o cátedras, discusiones en grupos pequeños, trabajos individuales, simulaciones, estudios de casos, juego de roles, actividades de aprendizaje experimental y trabajos colaborativos.

La Agencia para el Aseguramiento de la Calidad [QAA] de la educación superior en Reino Unido reconoce que la EDS contempla la adopción de enfoques de enseñanza y aprendizaje, como son una perspectiva interdisciplinar, un aprendizaje basado en la experiencia y en la interacción, la reflexión crítica, un aprendizaje significativo basado en el aprendizaje con problemas y situaciones de la vida real, y el aprendizaje participativo y entre pares (QAA, Higher Education Academy, 2014).

La adopción del enfoque de la EDS exige cambiar modelos de educación tradicionales que privilegian la formación disciplinar con muy poca flexibilidad. En cambio, sugiere modelos basados en la formación de competencias que permitan, junto con las propias de una disciplina, el egreso de futuros profesionales que puedan contribuir al desarrollo económico, social y ambiental. De acuerdo con Gradilla (2017), una educación basada en el desarrollo de competencias sustentables "permite a los estudiantes obtener los conocimientos, las habilidades y valores que reunirán en su vida personal y futuro profesional para enfrentar los retos de sostenibilidad" (p. 31).

Por lo tanto, la incorporación del enfoque de la EDS en el currículo representa un proceso que no está exento de tensiones entre las formas de comprender su alcance y su aplicación por parte de los docentes en su práctica académica. En otras palabras, esta forma 


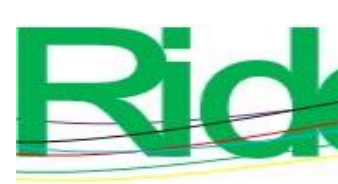

Revista Iberoamericana para la
Investigación y el Desarrollo Educativo
ISSN $2007-7467$

de educación alterna demanda un cúmulo de principios, conceptos y prácticas que pueden ser empleados para ayudar a estudiantes, docentes y gestores en la comprensión del desarrollo sustentable.

\section{Discusión}

Las universidades públicas del país, como organizaciones complejas, tienen el reto de comprender e implementar los principios de la sustentabilidad dentro del currículo universitario como parte de su compromiso con la Agenda 2030 y los objetivos para el desarrollo sustentable. Este reto implica conocer la forma en que son entendidos y usados por los distintos actores de la comunidad universitaria. Asimismo, y dado que el desarrollo sostenible es un concepto holístico que no atañe a una sola disciplina, se requiere la participación activa de todos los actores universitarios para facilitar su implementación.

En tal sentido, se debe tomar en cuenta que la estructuración actual que caracteriza a las universidades públicas del país — basada en la departamentalización del conocimientocontribuye a la fragmentación de la ciencia, realidad cuestionada por la EDS, la cual invita a la reconciliación de las distintas disciplinas para contribuir al diseño y desarrollo de nuevos modelos teórico-metodológicos que permitan dar respuestas a los desafíos que enfrenta el desarrollo sostenible.

Como ya se dijo, el enfoque de la EDS es un referente de carácter supranacional que surge en el consenso de los países, a pesar de sus diferencias económicas, sociales y ambientales, de ahí que los factores de contingencia obliguen a las organizaciones a su transformación y adaptación; por lo tanto, las decisiones supranacionales corresponderían a un factor de contingencia que afecta a las universidades públicas del país.

Por su parte, Olaskoaga, Marúm, Rosario y Pérez (2013) afirman que las instituciones de educación superior (IES) guardan una configuración estructural tradicional de tipo burocrática que obstaculiza los cambios requeridos para responder a los problemas del entorno. Por ende, los autores argumentan que el modelo que mejor encaja en el quehacer de las universidades es el que corresponde a la adhocracia, la cual recurre a la coordinación espontánea de los docentes para crear una cultura basada en el reconocimiento, lo que propicia la capacidad de aprendizaje e innovación.

De acuerdo con lo anterior, resulta imprescindible impulsar una gestión universitaria que se base en la participación del profesorado para facilitar la incorporación de la sustentabilidad en todos los programas de estudio. En efecto, en el contexto universitario el 


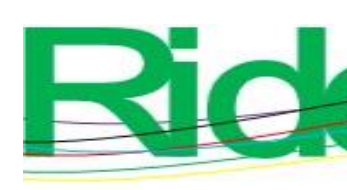

Revista Iberoamericana para la
Investigación y el Desarrollo Educativo
ISSN $2007-7467$

profesorado es un actor clave para desarrollar las competencias particulares de una disciplina, así como las generales, las cuales se reflejan en el desarrollo de un pensamiento sistémico y una visión a futuro. Por ello, para Cebrián (2020) “el apoyo y liderazgo institucional debe crear una comunidad de aprendizaje donde se identifiquen buenas prácticas existentes, se promueva el intercambio de recursos educativos y se disponga de apoyo y orientación por parte de expertos y facilitadores" (p. 111). En otras palabras, si bien la formación y actualización docente son un aspecto clave para desarrollar en el estudiantado las competencias requeridas por la EDS, también lo es su participación en el diseño de las estrategias institucionales para que las universidades logren contribuir con su misión y con el desarrollo sustentable.

En definitiva, el reto para la gestión universitaria es fomentar los programas de actualización y desarrollo docente, así como desarrollar en el profesorado el liderazgo que permita lograr la transformación del estudiante como un agente de cambio en la solución de los problemas medioambientales (Zúñiga-Sánchez, 2019).

\section{Conclusiones}

Establecer la perspectiva de la EDS en la educación superior es una tarea aún pendiente. Por ello, en este ensayo se tuvo el propósito de delinear aspectos cruciales que puedan servir de guía para gestores y tomadores de decisión de las políticas universitarias en el proceso de incorporar la perspectiva de la sustentabilidad en el currículo universitario. Para eso, sin embargo, se deben considerar las formas de estructuración que guardan las universidades públicas del país, dado que la EDS exige que estas instituciones actualicen su modelo educativo y académico para contemplar esta perspectiva, y con esto lograr una genuina formación interdisciplinaria que facilite el diálogo entre las disciplinas como condición necesaria para resolver los problemas que demanda el desarrollo sostenible. Justamente, una educación de calidad incluye que se apliquen metodologías propuestas por la EDS para garantizar la pertinencia de la educación superior con las sociedades del conocimiento.

Por último, como se expuso anteriormente, se necesita que el profesorado asuma un rol activo en el proceso de implementación de la perspectiva de la sustentabilidad. El reto de la gestión universitaria, en definitiva, es establecer mecanismos estructurales que promuevan 


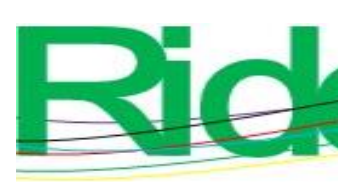

Revista Iberoamericana para la Investigación y el Desarrollo Educativo ISSN $2007-7467$

Antunes, A. y Gadotti, M. (2006). La ecopedagogía como la pedagogía indicada para el proceso de la Carta de la Tierra. En AA. VV. (2006), La Carta de la Tierra en acción. Hacia un mundo sostenible (pp. 141-143). Amsterdam: Kit Publishers.

Arbuthnott, K. D. (2009). Education for sustainable development beyond attitude change. International Journal of Sustainability in Higher Education, 10(2), 152-163.

Arias, M. A. (2010). Educación ambiental y sociedad civil en México: análisis de sus propuestas pedagógicas (tesis de doctorado). Madrid: Departamento de Ecología, Universidad Autónoma de Madrid.

Asociación Nacional de Universidades e Instituciones de Educación Superior [Anuies] y Secretaría de Desarrollo Urbano y Ecología [Sedesu) (1990). Formación ambiental. Órgano informativo del comité de la formación ambiental de las instituciones de educación superior mexicanas, 1(1), 1-24.

Batllori, A. (2008). La educación ambiental para la sustentabilidad: un reto para las universidades. México: UNAM.

Benayas, J., Alba, D. y Sánchez, S. (2002). La ambientalización de los campus universitarios: el caso de la Universidad Autónoma de Madrid. Ecosistemas, 11(3).

Berdugo, B. Z. (2013). Educación y aprendizaje desde la biopedagogía. Perspectivas de cambio para los procesos educativos. Caminos Educativos, 2, 103-111.

Bermejo, R. (2014). Del desarrollo sostenible según Brundtland a la sostenibilidad como biomimesis. Recuperado de http://publicaciones.hegoa.ehu.es/publications/315

Biasutti, M., De Baz, T. y Alshawa, H. (2016). Assessing the Infusion of Sustainability Principles into University Curricula. Journal of Teacher Education for Sustainability, $18(2), 21-40$.

Biasutti, M., Makrakis, V., Concina, E. and Frate, S. (2018). Educating academic staff to reorient curricula in ESD. International Journal of Sustainability in Higher Education, 19(1), 179-196. Doi: https://doi.org/10.1108/IJSHE-11-2016-0214

Bifani, P. (2013). Reflexiones irreverentes sobre educación ambiental. Jandiekua Revista Mexicana de Educación Ambiental, 1(1), 43-55.

Boada, M. y Toledo, V. (2003). El planeta, nuestro cuerpo. La ecología, el ambientalismo y la crisis de la modernidad. México: Fondo de Cultura Económica.

Bravo, M. T. (2012). Los planes ambientales en la educación superior de México. Construyendo sentidos de sustentabilidad (2002-2007) (1. a ed.). INE-SEMARNAT. Recuperado de http://www2.inecc.gob.mx/publicaciones/download/689.pdf 


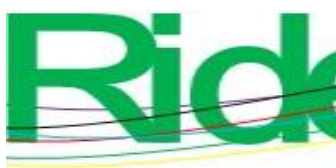

Revista Iberoamericana para la
Investigación y el Desarrollo Educativo
ISSN $2007-7467$

Bravo, M. T. (2008). La educación ambiental en México: visiones y proyecciones de actualidad. En Reyes-Escutia, F. y Bravo-Mercado, M. T. (coords.), Educación ambiental para la sustentabilidad en México. Aproximaciones conceptuales, metodológicas y prácticas (pp. 14-46). México: UNICACH. Recuperado de http://anea.org.mx/docs/EdAmbSustentabilidadMexico.pdf

Calixto, R., Herrera Reyes, L. y Hernández Guzmán, V. (2008). Ecología y medio ambiente (2. ${ }^{\mathrm{a}}$ ed.). Cengage Learning Editores.

Cañal, P., García, J. y Porlán, R. (1958). Ecología y escuela: teoría y práctica de la educación ambiental (2. ${ }^{\mathrm{a}}$ ed.). Laia.

Cebrián, G. (2020). La educación para el desarrollo sostenible en el currículum universitario: una investigación-acción cooperativa con profesorado. Revista Iberoamericana de Educación Superior, 11(30). Doi: https://doi.org/10.22201/iisue.20072872e.2020.30.590

Clark, B. (2001). The entrepreneurial university: New foundations for collegiality, autonomy, and achievement. Journal of the Programme on Institutional Management in Higher Education, 13(2), 9-23.

Coya, M. (2000). La ambientalización de la universidad. Un estudio sobre la formación ambiental de los estudiantes de la Universidad de Santiago Compostela y la política ambiental de la institución (tesis de doctorado). Universidad de Santiago Compostela.

Curiel, F., Peniche, S., Reyes, R. E., Alvarado, T. de J. y Hernández, G. (2005). Economía ambiental y desarrollo sustentable. Universidad de Guadalajara.

Dimas-Sánchez, P., Peña-Moscoso, A. O. y Herrán-Bocanegra, C. E. (2017). Ecopedagogía y buen vivir: los caminos de la sustentabilidad. Praxis, 13(1). Doi: https://doi.org/10.21676/23897856.2065

Fuentes, N. y González, H. (2016). Ambientalización del currículo universitario: un reto de la ecopedagogía. Rev. Fac. Cienc. Tecnol., (40), 310-339.

Gadotti, M. (2003). Pedagogía de la tierra y cultura de la sustentabilidad. Pedagogía Crítica, 2(2), 61-76.

González, E. J. y Arias, M. A. (2015). La investigación en educación ambiental para la sustentabilidad en México. 2002-2011. ANUIES.

González, E. J. y Garza, R. (2013). La educación superior frente al desafío de la sustentabilidad. Breve experiencia en la Universidad Autónoma Agraria Antonio 

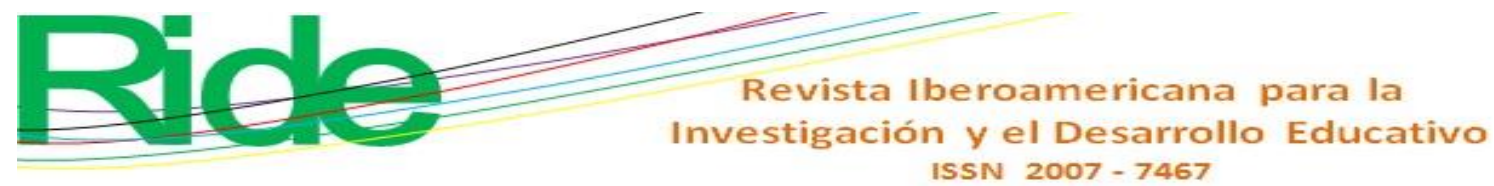

Narro. En La Educación Superior ante los Retos de la Sustentabilidad (1. ${ }^{a}$ ed.). UANL.

González-Gaudiano, E. J. y Puente-Quintanilla, J. C. (2010). El perfil de la educación ambiental en América Latina y el Caribe: un corte transversal en el marco del decenio de la educación para el desarrollo sustentable. Pesquisa em Educação Ambiental, 5(1), 27-45. Doi: https://doi.org/10.18675/2177-580X.vol5.n1.p27-45

Gradilla, M. (2017). Contribución de las disciplinas académicas al desarrollo de competencias para el desarrollo sostenible en los estudiantes: la perspectiva de los académicos (tesis de doctorado). CUCEA, Universidad de Guadalajara.

Guevara, J. (2013). El reto: simpatía paradigmática para una educación ambiental dúctil. En Fernández-Crispín, A. (ed.), La educación ambiental en México. Definir el campus y emprender el habitus. México: Benemérita Universidad Autónoma de Puebla.

Gutiérrez, B. E. y Martínez, M. C. (2010). El plan de acción para el desarrollo sustentable en las instituciones de educación superior. Escenarios posibles. Revista de la Educación Superior, 39(154), 111-132.

Gutiérrez, E. y González, E. (2010). De las teorías del desarrollo, al desarrollo sustentable: Construcción de un enfoque multidisciplinario. México: Siglo XXI.

Heidt, T. and Lamberton, G. (2011). Sustainability in the undergraduate and postgaduate business curriculum of a regional university: A critical perspective. Journal of Management and Organization, 17(5), 670-690.

Instituto de Investigación de Tecnología Educativa [Inite] (2004). Desarrollo sustentable (2. ${ }^{\text {a }}$ ed). Instituto de Investigación de Tecnología Educativa.

Junyent, M., Bonil, J. y Calafell, G. (2011). Evaluar la ambientalización curricular de los estudios superiores: un análisis de la red EDUSOST. Ensino Em Re-vista, 18(2), 323340.

Leal Filho, W. (ed.). (2015). Transformative Approaches to Sustainable Development at Universities. Springer International Publishing. Doi: https://doi.org/10.1007/978-3319-08837-2

Leff, E. (2002). Saber ambiental (2. ${ }^{a}$ ed.). México: Siglo XXI.

Lescano, J., Vegas, E., Collazos, H., Valdez, L. y Belaúnde, M. (2008). Planteamiento teórico y conceptual del desarrollo sostenible (informe Brundtland) "Nuestro futuro en común”. Universidad Nacional de la Amazona Peruana. 


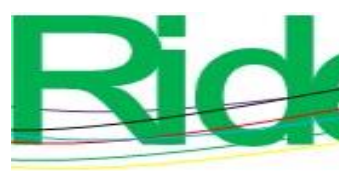

Revista Iberoamericana para la Investigación y el Desarrollo Educativo ISSN $2007-7467$

López, V. M. (2008). Sustentabilidad y desarrollo sustentable. Origen, precisiones conceptuales y metodología operativa. México: Trillas.

Lozano, R., Lukman, R., Lozano, F. J., Huisingh, D. and Lambrechts, W. (2013). Declarations for sustainability in higher education: Becoming better leaders, through addressing the university system. Journal of Cleaner Production, 48, 10-19. Doi: https://doi.org/10.1016/j.jclepro.2011.10.006

Matos, B. B. y Flores, M. A. (2016). Educación ambiental para el desarrollo sostenible del presente milenio (2. ${ }^{\mathrm{a}}$ ed.). Eco Ediciones.

Molano, A. C. y Herrera, J. F. (2014). La formación ambiental en la educación superior: una revisión necesaria. Recuperado de http://lunazul.ucaldas.edu.co/index.php?option=content $\&$ task=view \&id=955

Moreno, M. L., Barrientos, Z. y Johnson, H. (2009). Gestión ambiental en universidades públicas costarricenses: el ejemplo de "UNA-Campus sostenible". Revista Posgrado y Sociedad, 9(2), 81-124.

Noguera de Echeverri, A. (2013). Despliegues investigativos de su pensamiento en Educación Ambiental. En Fernández-Crispín, A. (ed.), La educación ambiental en México. Definir el campus y emprender el habitus (pp. 87-106). México: Benemérita Universidad Autónoma de Puebla.

Novo, M. (2003). La educación ambiental. Bases éticas, conceptuales y metodológicas (3. ${ }^{\text {a }}$ ed.). Universitas.

Novo, M. (2009). La educación ambiental, una genuina educación para el desarrollo sostenible. Revista de Educación, 195-217.

Olaskoaga, J., Marúm, E., Rosario, V. y Pérez, D. (2013). Universidades en movimiento. El debate acerca de la gestión de la calidad y las actitudes del profesorado ante las transformaciones universitarias. México: Anuies.

Pabón, R., Vargas, M. F., Rincón, A. y Garzón, G. (2005). Biopedagogía: sistematización de la estrategia educativa del Programa Desarrollo y Paz del Magdalena Medio. Centro de Investigación y Educación Popular.

Paniagua, Á. y Moyano, E. (1998). Medio ambiente, desarrollo sostenible y escalas de sustentabilidad. Reis, 83, 151-175. Doi: https://doi.org/10.2307/40184124

Patarroyo, L. E., Guerrero, L. G., Rincón, A. y Vargas, M. F. (2011). Biopedagogía.

Quadri de la Torre, G. (2006). Políticas públicas. Sustentabilidad y medio ambiente. México: Miguel Ángel Porrúa. 


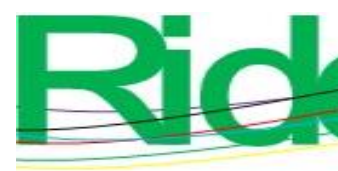

Revista Iberoamericana para la
Investigación y el Desarrollo Educativo
ISSN $2007-7467$

Ramírez, A., Sánchez, J. M. y García, A. (2004). El desarrollo sustentable: interpretación y análisis. Revista del Centro de Investigación, 6(21), 55-59.

Ramírez, D. y Ramírez, J. (2014). Derecho ambiental y desarrollo sustentable (2. ${ }^{\mathrm{a}}$ ed.). México: Porrúa.

Romero, Y. (2012). El bisturí de oro. Una reflexión sobre biopedagogía y antropología de la medicina, la salud y el chamanismo. Tabula Rasa, 16, 283-293.

Ruiz, R., Martínez, R. y Valladares, L. (2010). Innovación en la educación superior. Hacia las sociedades del conocimiento. México: Fondo de Cultura Económica/UNAM.

Sauvé, L. (2010). Educación científica y educación ambiental: un cruce fecundo. Enseñanza de las Ciencias, 28(1), 5-18.

Sauvé, L. (2013). La dimensión política de la educación ambiental: un cierto vértigo. En Fernández-Crispín, A. (ed.), La educación ambiental en México. Definir el campus y emprender el habitus. Benemérita Universidad Autónoma de Puebla.

Secretaría de Medio Ambiente y Recursos Naturales [Semarnat]. (2006). Estrategia de educación ambiental para la sustentabilidad en México. Gobierno de la República. Recuperado de http://www2.inecc.gob.mx/publicaciones/consultaPublicacion.html?id_pub=579

Svanström, M., Lozano-García, F. J. y Rowe, D. (2008). Learning outcomes for sustainable development in higher education. International Journal of Sustainability in Higher Education, 9(3), 339-351. Doi: https://doi.org/10.1108/14676370810885925

United Nations Educational, Scientific and Cultural Organization [Unesco]. (1977). Conferencia intergubernamental sobre Educación Ambiental. Tbilisi (URSS) 14-26 de octubre $\quad 1977 . \quad$ Retrieved from http://unesdoc.unesco.org/images/0003/000327/032763sb.pdf

United Nations Educational, Scientific and Cultural Organization [Unesco]. (2005). Hacia las sociedades del Conocimiento. Unesco. Recuperado de http://unesdoc.unesco.org/images/0014/001419/141908s.pdf

United Nations Educational, Scientific and Cultural Organization [Unesco] y Pnuma (1975). Seminario internacional de educación ambiental. Recuperado de http://unesdoc.unesco.org/images/0002/000276/027608SB.pdf

Wright, T. S. A. (2002). Definitions and Frameworks for Environmental Sustainability in Higher Education. International Journal of Sustainability in Higher Education, 3(3), 203-220. 

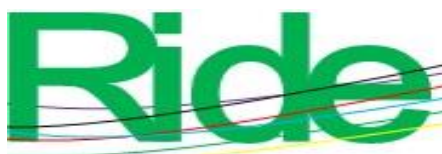

Revista Iberoamericana para la
Investigación y el Desarrollo Educativo ISSN $2007-7467$

Zingaretti, H. E. (2008). La ecopedagogía y la formación de los niños. Universidad Nacional de Cuyo. Recuperado de https://feeye.uncuyo.edu.ar/web/X-CNREDUEI/eje3/Zingaretti.pdf

Zúñiga-Sánchez, O. (2019). Estilo de liderazgo del profesorado: el caso de la licenciatura en ingeniería en Ciencias Computacionales del Centro Universitario de Tonalá, Universidad de Guadalajara. En Madrigal Torres, B. E. y Ramírez Mata, M. (comps.), La ética y el liderazgo en las instituciones latinoamericanas de educación superior en la cuarta revolución industrial (pp. 79-97). Fondo Editorial Universitario. 